\title{
GENERATING FUNCTIONS FOR THE JACOBI POLYNOMIAL
}

\author{
M. E. COHEN
}

\begin{abstract}
Two theorems are proved with the aid of operator and series iteration methods. Special cases appear to give new and known generating functions for the Jacobi polynomial.
\end{abstract}

I. Introduction. Two new general theorems are presented for double series using a generalization of the operators in [5]. This approach differs from usual procedures adopted by previous workers in that it does not appeal to the Lagrange theorem.

Theorem 2 yields a generating function for the Jacobi polynomial

$$
\begin{aligned}
\sum_{k=0}^{\infty} \frac{t^{k}}{(1-s k / b)} P_{k}^{(a, b-(s+1) k)}(x)= & \frac{(1-z)^{a+1}[2-(1-x)(1-z)]^{b}}{(1+x)^{b}} \\
& \cdot{ }_{2} F_{1}\left[\begin{array}{c}
a+1,1 ; \\
-b / s+1 ;
\end{array}\right]
\end{aligned}
$$

where

$$
t=(-2 z)[2-(1-x)(1-z)]^{s} /(1-z)(1+x)^{s+1},
$$

and $s$ is an arbitrary complex number.

(1.1) appears to be new. A special case of interest occurs when $b=-a s$ in (1.1), giving

$$
\begin{aligned}
\sum_{k=0}^{\infty} \frac{t^{k}}{(1+k / a)} P_{k}^{(a,-a s-(s+1) k)}(x) & \\
& =(1+x)^{a s}(1-z)^{a} /[2-(1-x)(1-z)]^{a s} .
\end{aligned}
$$

For $s=-1,(1.1)$ and (1.2) reduce to the particular case of the known Brafman generating function [1] for the Jacobi and Gegenbauer polynomials, respectively, given by his equations (12) and (17).

A special case of Theorem 1 is

$$
\begin{aligned}
\sum_{k=0}^{\infty} t^{k} P_{k}^{(a, b-(s+1) k)}(x) & \\
= & \frac{(1-z)^{a+1}[2-(1-x)(1-z)]^{b+1}}{(1+x)^{b}[2-(1-x)(1-z)(1-s z)]}
\end{aligned}
$$

Received by the editors February 10, 1975 and, in revised form, September 15, 1975.

AMS (MOS) subject classifications (1970). Primary 33A65, 33A30.

Key words and phrases. Generating function, series iteration, differential operators, Jacobi polynomial. 
where $t$ is defined as in (1.1). An elegant generalization of (1.3) was obtained by Srivastava [7]. These results extend Jacobi's generating function [10, p. 68].

$$
\sum_{k=0}^{\infty} t^{k} P_{k}^{(a, b)}(x)=2^{a+b} \rho^{-1}(1+t+\rho)^{-b}(1-t+\rho)^{-a}
$$

where $\rho=\left(1-2 x t+t^{2}\right)^{1 / 2}$.

Brown [2] and Feldheim [6] obtained new generating functions for the Jacobi polynomial, which were extended by Srivastava [9], to give

$$
\begin{aligned}
\sum_{n=0}^{\infty} P_{n}^{(\alpha-n, \beta-(b+1) n)}(x) t^{n} & \\
= & (1+w)^{-\alpha-\beta}(1+b w)^{-1}[1+2 w /(1-x)]^{\alpha}
\end{aligned}
$$

where $w=\frac{1}{2}(1-x)(1+w)^{b+1}$, and

$$
\begin{aligned}
\sum_{n=0}^{\infty} P_{n}^{(\alpha+b n, \beta-(b+1) n)}(x) t^{n} & \\
= & (1+v)^{\alpha+1}(1-b v)^{-1}\left[1-\frac{1}{2}(x-1) v\right]^{-\alpha-\beta-1}
\end{aligned}
$$

where $v=t(1+v)^{b+1}$.

It was pointed out by the referee that equation (9), the main result of Srivastava [9], appears to have first been communicated by Brown [3].

(1.5) and (1.6) can also be deduced from Theorem 1.

Carlitz [4], Srivastava [8], Zeitlin [12], Verma [11], Cohen [5], and others have subsequently extended (1.5) and (1.6) to other systems of polynomials.

II.

THEOREM 1. For $r, s, \alpha$, and $\beta$ any arbitrary complex numbers,

$$
\begin{gathered}
\sum_{k=0}^{\infty} \sum_{p=0}^{\infty} \frac{(-z)^{k}(-y)^{p}(\alpha+1)_{k+r p}(\beta+1)_{p+s k}}{k ! p !(\alpha+1)_{r p}(\beta+1)_{s k}(1-z)^{k+r p}(1-y)^{p+s k}} \\
=(1-z)^{\alpha+1}(1-y)^{\beta+1} /(1-r s y z)
\end{gathered}
$$

where

$$
\begin{aligned}
(a)_{n} & \equiv \Gamma(a+n) / \Gamma(a) \\
& \equiv(a)(a+1) \cdots(a+n-1) \quad \text { for } n \text { a positive integer } \\
& \equiv 1 \quad \text { for } n=0,
\end{aligned}
$$

and $|y|<1,|z|<1$, and $|r s y z|<1$.

Proof. Consider the expression

$$
\sum_{n=0}^{\infty} \sum_{m=0}^{\infty} \frac{z^{n} y^{m}}{n ! m !} D^{n}\left\{x^{n+\alpha}\left(1-x^{r}\right)^{m}\right\} D^{m}\left\{x^{m+\beta}\left(1-x^{s}\right)^{n}\right\}
$$

Putting the operators in polynomial form, (2.2) reduces to 


$$
\sum_{n=0}^{\infty} \sum_{m=0}^{\infty} \frac{z^{n} y^{m}}{n ! m !}
$$

$$
\begin{aligned}
\cdot \sum_{k=0}^{n} \sum_{p=0}^{m} \frac{(-n)_{k}(-m)_{p} \Gamma(n+\alpha+1+r p) \Gamma(m+\beta+1+s k)}{k ! p ! \Gamma(\alpha+1+r p) \Gamma(\beta+1+s k)} \\
\cdot x^{\alpha+\beta+r p+s k} .
\end{aligned}
$$

Take (2.2) and (2.3) at $x=1$. In (2.2) only $n=m$ contributes, reducing it to

$$
\sum_{n=0}^{\infty}(r s y z)^{n}=(1-r s y z)^{-1} \text {. }
$$

Applying the series transformation

$$
\begin{aligned}
\sum_{n=0}^{\infty} \sum_{m=0}^{\infty} \sum_{p=0}^{m} \sum_{k=0}^{n} f(n, m, k, p) \\
=\sum_{n=0}^{\infty} \sum_{m=0}^{\infty} \sum_{p=0}^{\infty} \sum_{k=0}^{\infty} f(n+k, m+p, k, p)
\end{aligned}
$$

to (2.3), taken at $x=1$, using

$$
\begin{aligned}
\sum_{n=0}^{\infty} \frac{z^{n}(\alpha+1+k+r p)_{n}}{n !} & =\frac{1}{(1-z)^{\alpha+1+k+p}}, \\
\sum_{n=0}^{\infty} \frac{y^{m}(\beta+1+p+s k)_{m}}{m !} & =\frac{1}{(1-y)^{\beta+1+p+s k}}
\end{aligned}
$$

and algebraic manipulation proves (2.1).

COROLlary 1. We shall indicate here the procedure in obtaining the special case (1.3) from Theorem 1. Taking $r=1$ in (2.1), one has

$$
\begin{aligned}
& \sum_{k=0}^{\infty} \frac{(-z)^{k}(\alpha+1)_{k}}{k !(1-z)^{k}(1-y)^{s k}} \sum_{p=0}^{\infty} \frac{(\alpha+1+k)_{p}(\beta+1+s k)_{p}}{(\alpha+1)_{p} p !}\left[\frac{-y}{(1-z)(1-y)}\right]^{p} \\
& =\sum_{k=0}^{\infty} \frac{(-z)^{k}(\alpha+1)_{k}}{k !(1-z)^{k}(1-y)^{s k}}{ }_{2} F_{1}\left[\begin{array}{cc}
\alpha+1+k, \beta+1+s k ; & \frac{-y}{(1-z)(1-y)} \\
\alpha+1 ; &
\end{array}\right] \\
& =(1-z)^{\alpha+1}(1-y)^{\beta+1} /(1-s y z) .
\end{aligned}
$$

Using Gauss' transformation for the hypergeometric function

$$
{ }_{2} F_{1}[a, b ; c ; x]=(1-x)^{c-a-b}{ }_{2} F_{1}[c-a, c-b ; c ; x],
$$

letting $(-y /(1-y))=(1-z)(1-x) / 2, \alpha=a, \beta=-1-b$, and using the Jacobi representation

$$
P_{n}^{(e, f)}(x)=\frac{(e+1)_{n}}{n !}{ }_{2} F_{1}\left[\begin{array}{cc}
-n, e+f+1+n ; & \frac{1-x}{2} \\
e+1 ; &
\end{array}\right]
$$

gives (1.3). 
Corollary 2. Using Theorem 1, (1.5) and (1.6) can be derived. Taking $r=-1$, using transformation (2.8), and variable changes

$$
y^{\prime}=-y(1-z) /(1-y) \text {, }
$$

one obtains

$$
\begin{aligned}
\sum_{k=0}^{\infty} \frac{(\alpha+1)_{k}(-z)^{k}\left(1-z-y^{\prime}\right)^{s k}}{k !\left[(1-z)\left(1-y^{\prime}\right)\right]^{(s+1) k}} & \\
& \cdot{ }_{2} F_{1}\left[\begin{array}{cr}
-k,-\alpha-\beta-1-k-s k ; & y^{\prime} \\
\alpha-k ; &
\end{array} \quad(1-z)^{\alpha+\beta+2}\left(1-y^{\prime}\right)^{\beta+1} /\left(1-z-y^{\prime}\right)^{\beta}\left(1-z-y^{\prime}-s y^{\prime} z\right) .\right.
\end{aligned}
$$

Equation (2.10) is essentially Srivastava's equation 8 [9]. Note

$$
\sum_{n=0}^{k} f(n, k)=\sum_{n=0}^{k} f(k-n, k) .
$$

Hence, the appropriate hypergeometric representations for the Jacobi polynomial and variable changes give (1.5) and (1.6).

THEOREM 2. For $s, \alpha$, and $\beta$ any arbitrary complex numbers,

$$
\begin{gathered}
\sum_{p=0}^{\infty} \sum_{k=0}^{\infty} \frac{(-y)^{p}(-z)^{k}(\alpha)_{s k+p}(\beta+1)_{k+p}}{k ! p !(\alpha+1)_{s k}(\beta+1)_{p}(1-y)^{p+s k}(1-z)^{k+p}} \\
=(1-y)^{\alpha}(1-z)^{\beta+1}{ }_{2} F_{1}\left[\begin{array}{cc}
1, \beta+1 ; & \\
\alpha / s+1 ; & z
\end{array}\right]
\end{gathered}
$$

where $|y|$ and $|z|<1$.

Proof. Using (2.2) and (2.3), one has

$$
\begin{gathered}
D^{n}\left\{x^{n+\alpha}\left(1-x^{s}\right)^{m}\right\} D^{m}\left\{x^{m+\beta}(1-x)^{n}\right\} \\
=\sum_{k=0}^{m} \sum_{p=0}^{n} \frac{(-n)_{p}(-m)_{k} \Gamma(n+\alpha+s k+1) \Gamma(m+\beta+1+p)}{k ! p ! \Gamma(\alpha+1+s k) \Gamma(\beta+1+p)} \\
\cdot x^{\alpha+\beta+s k+p} .
\end{gathered}
$$

Multiplying both sides of (2.12) by $x^{-\beta-1}$, and integrating between 0 and 1 , we can deduce, using integration by parts, the result

$$
\begin{gathered}
\sum_{k=0}^{m} \sum_{p=0}^{n} \frac{(-n)_{p}(-m)_{k} \Gamma(n+\alpha+s k+1) \Gamma(m+\beta+1+p)}{k ! p ! \Gamma(\alpha+1+s k) \Gamma(\beta+1+p) \Gamma(\alpha+s k+p)} \\
=n ! m !(\beta+1)_{m} /(\alpha)(\alpha / s+1)_{m} .
\end{gathered}
$$

Multiplying both sides of (2.13) by $y^{n} z^{m} / n ! m$ ! and summing over $n$ and $m$ from 0 to $\infty$, one obtains, following the procedure as in Theorem 1, (2.11). 
COROllary 1. We derive the special case (1.1) from Theorem 2. Express (2.11) as

$$
\begin{aligned}
& \sum_{k=0}^{\infty} \frac{(-z)^{k}(\alpha)_{s k}(\beta+1)_{k}}{k !(\alpha+1)_{s k}(1-y)^{s k}(1-z)^{k}} \\
& \cdot{ }_{2} F_{1}\left[\begin{array}{cc}
\alpha+s k, \beta+1+k ; & -y \\
\beta+1 ; &
\end{array}\right] \\
& =(1-y)^{\alpha}(1-z)^{\beta+1}{ }_{2} F_{1}\left[\begin{array}{ll}
1, \beta+1 ; \\
\alpha / s+1 ;
\end{array}\right] .
\end{aligned}
$$

Using the transformation (2.8), letting

$$
\alpha=-b, \quad \beta=a, \quad y=(1-x)(1-z) /((1-x)(1-z)-2),
$$

and the Jacobi representation (2.9) gives (1.1).

Corollary 2. Proceeding as in Corollary 2 of Theorem 1, one may obtain the appropriate generating function for the Jacobi polynomial, which is a generalization of Brown's second and fifth equations in [2, p. 266], not generalized by Srivastava. See Cohen [5] for this result and generalization.

\section{REFERENCES}

1. F. Brafman, Generating functions of Jacobi and related polynomials, Proc. Amer. Math. Soc. 2 (1951), 942-949. MR 13, 649.

2. J. W. Brown, New generating functions for classical polynomials, Proc. Amer. Math. Soc. 21 (1969), 263-268. MR 38 \#4734.

3. , New generating functions for classical polynomials, Notices Amer. Math. Soc. 16 (1969), 412. Abstract \#69T-B29.

4. L. Carlitz, Some generating functions for Laguerre polynomials, Duke Math. J. 35 (1968), 825-827. MR 39 \# 1700.

5. M. E. Cohen, On expansion problems: New classes of formulae for the classical functions, SIAM J. Math. Anal. 7 (1976).

6. E. Feldheim, Relations entre les polynomes de Jacobi, Laguerre, et Hermite, Acta Math. 75 (1942), 117-138. MR 7, 65.

7. H. M. Srivastava, New generating functions for Jacobi and related polynomials, J. Math. Anal. Appl. 41 (1973), 748-752.

8., A class of generating functions for generalized hypergeometric polynomials, J. Math. Anal. Appl. 35 (1971), 230-235. MR 43 \#3509.

9. , Generating functions for Jacobi and Laguerre polynomials, Proc. Amer. Math. Soc. 23 (1969), 590-595. MR 40 \#2935.

10. G. Szegö, Orthogonal polynomials, rev. ed., Amer. Math. Soc. Colloq. Publ., vol. 23, Amer. Math. Soc., Providence, R. I., 1959. MR 21 \# 5029.

11. A. Verma, On generating functions of classical polynomials, Proc. Amer. Math. Soc. 46 (1974), 73-76. MR 49 \#9276.

12. D. Zeitlin, $A$ new class of generating functions for hypergeometric polynomials, Proc. Amer. Math. Soc. 25 (1970), 405-412. MR 41 \#8719.

Department of Mathematics, California State University, Fresno, California 93740 